\title{
Valoración de la aptitud psicomotora y la inteligencia requerida para conducir en los Centros de Reconocimiento de Conductores
}

\author{
Assessment of the psychomotor aptitude and \\ intelligence required in driving in the Drivers \\ evaluation centres
}

\author{
M. OZCOIDI* \\ M. R. SANZ* \\ C. CIVERA*** \\ L. MONTORO**
}

\begin{abstract}
RESUMEN
Objetivos, análisis de los aspectos considerados en la valoración de las aptitudes perceptivo-motoras y la inteligencia según el Modelo Español de reconocimiento de conductores. Material y Métodos, mediante la observación de variables analizadas en las normativas aplicadas desde que se inicia la locomoción en España y los protocolos actuales de valoración recomendados por la Dirección General de Tráfico y El Ministerio de Sanidad. Conclusiones, las variables consideradas han servido a los modelos preventivos de cada época, actualmente pretenden atender a las necesidades de prevención de los hábitos sociales actuales. Los aspectos valorados, apenas han sufrido variaciones, en los últimos años. El sistema de evaluación y registro de datos evolucionó con la normalización de los aparatos psicotécnicos en 1988, permaneciendo sin modificaciones hasta la actualidad. La actuación protocolizada es sin duda un sistema eficaz para determinar la actitud para conducir
\end{abstract}

\footnotetext{
* Creme Federación de Asociaciones de Centros de Reconocimientos Médicos.

** Instituto de Tráfico y Seguridad Vial (INTRAS) Universidad de Valencia (UVEG).

*** Cátedra de Prevención de Riesgos en los Comportamientos Viales. Mutua Madrileña-UVEG.
} 
Valoración de la aptitud psicomotora y la inteligencia requerida para conducir en los Centros de Reconocimiento...

\section{PALABRAS CLAVE}

Condiciones psicofisicas, Aptitudes perceptivo-motoras, Variables psicométricas, La inteligencia en la conducción.

\section{ABSTRACT}

Goals, analysis of the aspects on intelligence and perceptive-motor aptitudes according to the Spanish Driver model test. Material and methods, through observation of the analysed variables of the applied norm since locomotion started in Spain and the current protocols of evaluation recommended by the Direction General of Traffic and The Ministry of Health. Conclusions, the considerated variables have served the preventive models of each time period, currently their goal is to attend the prevention needs of the current social habits. The evaluated aspects have hardly changed in the last few years. The evaluation system and data collection have being developed with the standardisation of psycotechnical devices in 1988. Remaining unchanged until the present. The protocolized intervention is without question an effective system to determine the driving attitude.

\section{KEY WORDS}

Psychophysical conditions, Perceptive-motor aptitudes, Psychometric variables, Intelligence level to drive. 


\section{INTRODUCCIÓN}

España posee una larga experiencia en evaluación médico-psicológica de conductores (Serra y Tortosa, 2003; Tortosa y Montoro, 2002), así como una singularidad en el contexto Europeo (Tortosa, Barjonet, Civera y Montgoro, 2003). El modelo por el que nos regimos (Tortosa, Civera y Pastor, 2001), se basa en la existencia de unos Centros de Reconocimiento de Conductores (CRC) cuyo funcionamiento se inició en el año 1982 mediante el Real Decreto 1467/1982 de 28 de mayo (BOE 160 de 6 -VII- 82). Pero el informe de aptitud psico-físca para la obtención y renovación del permiso de conducir era ya preceptivo en nuestro país desde el año 1934 (Gaceta de Madrid, 269 de 26-IX-34) Y el certificado médico de aptitudes fisicas desde el año1900 ${ }^{1}$.

El Real Decreto 1467/82 de 28 de Mayo, establece la valoración psicotécnica, solamente para conductores profesionales. Posteriormente, el Real Decreto 2272/85 de 4 de diciembre (BOE 294 de 9-XII-85), amplía la valoración de las aptitudes perceptivo- motoras a todos los conductores.

La Orden Ministerial de 13 de mayo de 1986 (B.O.E. 121 de 21-V-86), establece los requisitos de autorización y funcionamiento de los C R C, y en su artículo séptimo, refiere: la Dirección General de Tráfico (DGT) debe crear "una Comisión Central para la debida uniformidad y normalización de los instrumentos y materiales a utilizar por todos los CRC en sus exploraciones psicológicas a los conductores. Esta Comisión de Normalización de equipos psicotécnicos se creó por resolución de la DGT. de 14-VI1986 (B.O.E. 222 de 16-IX-86). Unos meses después, una nueva resolución de la DGT. (B.O.E. 68 de 20-III-87) determinaba la información a suministrar por los fabricantes de los equipos psicotécnicos para su normalización. Hasta que, finalmente, la DGT. prorrogó hasta el 1 de octubre de 1988 la normalización de equipos (B.O.E. 279 de 21-XI-1987).

Fruto de todo este proceso fue la creación y aceptación de las Baterías de Test psicotécnicos para Centros de Reconocimiento de Conductores (CRC).

La última actualización de la normativa aparece en el anexo IV del Real Decreto $1598 / 2004$ de 2 de Julio (BOE 173 de 19 -VII- 04), sin variaciones sustanciales con respecto a la anterior.

La evaluación médico-psicológica de conductores es una actividad interdisciplinar. Y supone una medida preventiva básica, con importante responsabilidad en el control y evaluación de conductores con factores de riesgo asociados a los hábitos de conducta al conducir y su estado de salud.

Los profesionales que trabajan en los CRC, deben: decidir si una persona se encuentra en condiciones de conducir, aconsejar en qué condiciones deben llevar a cabo la conducción, y dar respuesta a situaciones que puedan afectar, en un momento dado, a su capacidad para realizar una conducción segura (Montoro y cols 2000).

En la valoración psicofisica de los conductores, se consideran, desde la normativa de 1982 y hasta la actualidad, 13 apartados (Tabla 1), en los que se recogen: aspectos, enfermedades y deficiencias de potencial riesgo vial que deben ser descartadas en los conductores.

\footnotetext{
${ }^{1}$ Puede consultarse en la Colección Legislativa de España, tomo VII, vol. 3º de 1900, Madrid, pág. 386.
} 
Valoración de la aptitud psicomotora y la inteligencia requerida para conducir en los Centros de Reconocimiento...

Las posibles deficiencias encontradas en cada apartado, pueden ser causa de: denegación, restricción y/o adaptación (espejos retrovisores, dirección asistida,. etc.) en el permiso de conducir (Tabla 1).

La determinación de la aptitud para conducir y si procede el establecimiento de condicionantes (restricciones, adaptaciones, limitaciones,.etc.), en el permiso de conducir, en ocasiones, no está acotada por la normativa, que deja a criterio facultativo esta valoración. Razón por la que los profesionales de los CRC deben seguir unos criterios de valoración uniformes para evitar discrepancias ante diferentes criterios en CRC diferentes.

\section{OBJETIVOS}

Analizar los aspectos considerados en la valoración de las aptitudes perceptivomotoras y la inteligencia a través de las normativas que han ido sucediéndose a lo largo de la historia de la locomoción en España

Analizar el sistema actual de valoración, según el Modelo Español de reconocimiento de conductores.

\section{MATERIAL Y MÉTODOS}

Determinar la aptitud para conducir en aspectos sujetos a medidas regladas, supone una tarea fácilmente asumible por el profesional, cuando las variables a a determinar pueden valorarse mediante test. Pero valorar los aspectos cuyas variables no pueden determinarse por aplicación de test, y deben descartarse para establecer la normalidad de las aptitudes perceptivo-motoras requiere la utilización de otras herramientas profesionales.

En le presente artículo, consideramos como bases de actuación de los profesio-

Tabla 1.- Apartados considerados en la valoración de los conductores (columna de la izquierda y posibles restricciones y adaptaciones en función del déficit hallado (resto de columnas).

\begin{tabular}{|c|c|c|c|c|c|}
\hline & & ADAP & $\begin{array}{l}\text { VL } \\
(05.04)\end{array}$ & $\begin{array}{l}\text { PV } \\
\text { (104) }\end{array}$ & $\begin{array}{l}\text { OTROS } \\
\text { OE }\end{array}$ \\
\hline 1 & A.VISUAL & $\mathrm{x}$ & $\mathrm{x}$ & $\mathbf{x}$ & $\mathbf{x}$ \\
\hline 2 & A.AUDITIVA & $\mathrm{x}$ & & $\mathrm{x}$ & $\mathrm{x}$ \\
\hline 3 & MOTILIDAD & $\mathrm{x}$ & $\mathrm{x}$ & $\mathrm{x}$ & $\mathrm{x}$ \\
\hline 4 & CARDIOVS & & & $\mathrm{x}$ & $\mathrm{x}$ \\
\hline 5 & HEMATOLG & & & $x$ & $\mathrm{x}$ \\
\hline 6 & RENAL & & & $\mathrm{x}$ & $\mathrm{x}$ \\
\hline 7 & RESPT & & & $\mathrm{x}$ & $\mathrm{x}$ \\
\hline 8 & METAB & & & $\mathrm{x}$ & $\mathrm{x}$ \\
\hline 9 & NERVIOSO & & & $\mathrm{x}$ & $\mathrm{X}$ \\
\hline 10 & MENTAL & & & $\mathrm{x}$ & $\begin{array}{l}\times \\
\times 05.03 \text { radio delimitado pasajeros } \\
05\end{array}$ \\
\hline 11 & ALC Y SUST & & & $\mathrm{x}$ & $\begin{array}{l}\times 55.04 \text { velocidad limitada } \\
05.05 \text { acompañado de otro conduct }\end{array}$ \\
\hline & PERC-MOT & $\mathbf{x}$ & $\mathrm{x}$ & $\mathrm{x}$ & $\begin{array}{l}05.06 \text { sin remolque } \\
05.07 \text { no permitida en autopista }\end{array}$ \\
\hline & OTRAS & & & $\mathbf{x}$ & x 05.08 exclusión de alcohol \\
\hline
\end{tabular}


nales de los CRC los protocolos de exploración para Centros de Reconocimiento de conductores propuestos en:

- Guía para la historia clínica básica (Varios 2002).

- Protocolo de Exploración Específica Médico-Psicológica en centros de reconocimiento de conductores. Guía para la Historia Clínica Específica (2003).

- Protocolo de Exploración médicopsicológica para Centros de Reconocimiento de Conductores. Ministerio de Sanidad-Dirección General de Tráfico(2007 ).

A través del análisis de estos protocolos proponemos la valoración actual de cada una de las variables contempladas en el apartado de la normativa correspondiente a la aptitud perceptivo motora para conducir.

\section{CONCLUSIONES}

Del análisis de las normativas, observamos: que históricamente para valorar las alteraciones perceptivo-motoras, se han considerado las variables: atenciónconcentración, velocidad de anticipación, coordinación bimanual vIsomotora, tiempo de reacción simple, tiempos de reacciones múltiples, inteligencia, capacidad de aprendizaje psicomotor, personalidad y estilos cognitivos (Figura 1).

Estás variables se han ido introduciendo, en fecha y tipo de permiso de conducir (B: PERMISOS NO PROFESIONALES), (C: PERMISOS PROFESIONALES) como se muestra en las columnas de la figura 1 (Ozcoidi 2005)

Actualmente las herramienta que unifican la valoración de La Aptitud Perceptivo Motora y la Inteligencia, están recogidas en protocolos(Varios 2002, (Varios

Figura 1. Variables Psicológicas que se han considerado en la valoración de conductores en el Modelo Español, por tipos de permisos (B no profesionales, C profesionales)y en los años en los que ha sido preceptiva su valoración

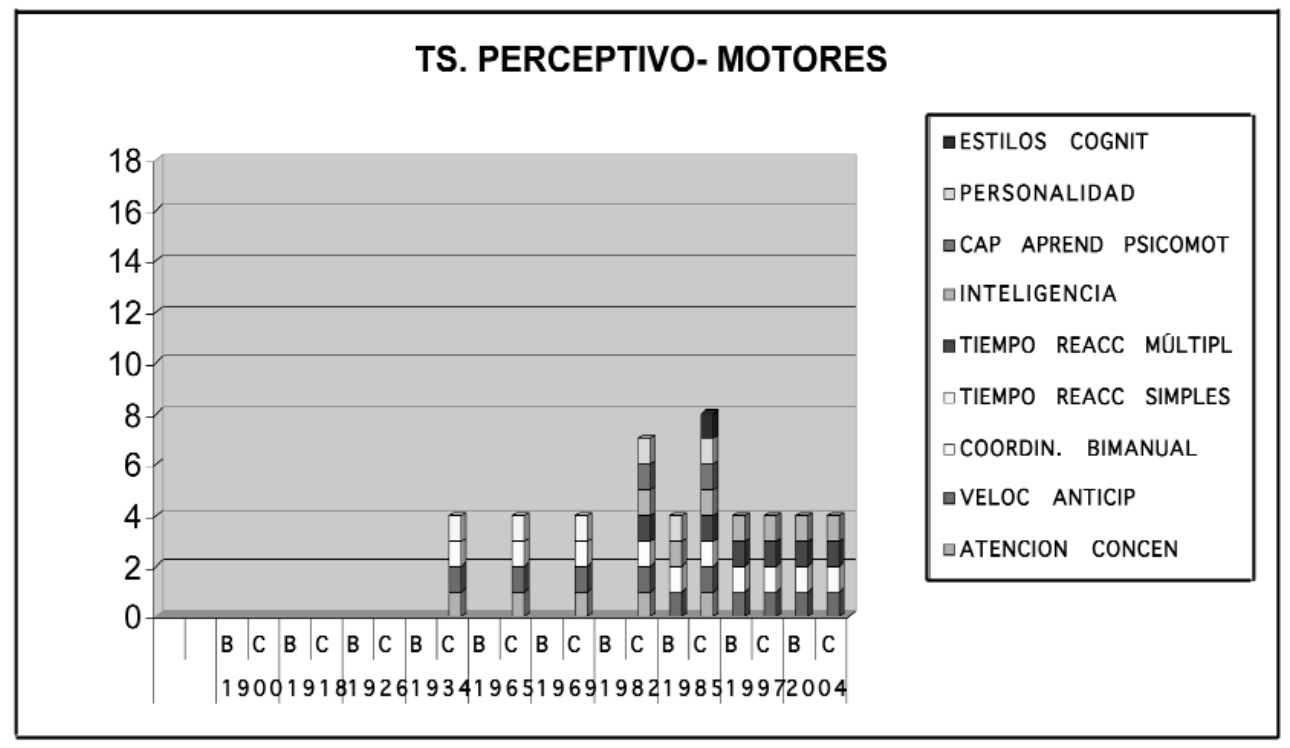


Valoración de la aptitud psicomotora y la inteligencia requerida para conducir en los Centros de Reconocimiento..

2003), (Varios 2007). En los que esquemáticamente se siguen las pautas siguientes:

\section{- EXPLORACION BASICA DE PSICOLOGIA}

Que debe comprender al menos, la valoración de:

\section{- LA PRIMERA IMPRESIÓN \\ - APTITUD PERCEPTIVO MOTORA \\ - INTELIGENCIA \\ - TRASTORNOS MENTALES Y DE CONDUCTA \\ - EXPLORACIÓN DE ÁREAS COG- NITIVAS}

\section{LA PRIMERA IMPRESIÓN}

\begin{tabular}{|l|l|l|}
\hline Aspecto Personal & & \\
\hline Habla & & \\
\hline Gestos & & \\
\hline Conducta & & \\
\hline
\end{tabular}

La observación de las características que el aspirante presenta a simple vista, nos dará, una información fundamental para enfocar la entrevista y decidir el tipo de exploración conveniente.

El grado de autocuidado, la limpieza, el cuidado en el vestir, la concordancia entre el aspecto y la información verbal que nos facilita; la fluidez, volumen, cla- ridad y expresividad del habla; una gesticulación adecuada a la información que nos facilita, y la actitud gestual general (postura corporal o el contacto visual); así como, el mantenimiento de una conducta adecuada, atención y colaboración suficientes, ausencia de agresividad, etc., son factores en los que centraremos nuestra atención.

\section{APTITUDES PERCEPTIVO-MOTORAS}

\begin{tabular}{|c|c|c|c|c|}
\hline & \multicolumn{3}{|c|}{ Normal } & Alterada \\
\hline \multicolumn{5}{|l|}{ Calidad ejecución } \\
\hline \multicolumn{5}{|l|}{ Comprensión instrucciones } \\
\hline \multicolumn{5}{|l|}{ Atención, concentración } \\
\hline \multicolumn{5}{|l|}{ Nerviosismo-Tranquilidad } \\
\hline & Normal & Alterada & PC TI & Pr-Re \\
\hline \multicolumn{5}{|l|}{ VELOCIDADDE ANTICIPACIÓN } \\
\hline & Normal & Alterada & PC T & PC NT \\
\hline \multicolumn{5}{|l|}{ COORDINACIÓN BIMANUAL } \\
\hline & Normal & Alterada & $\mathrm{PC} T$ & PC ER \\
\hline TIEMPOS REAC. MÚLTIPLES & & & & \\
\hline
\end{tabular}

La aptitud preceptivo-motora se evalúa con los instrumentos informatizados con que cuentan los CRC; se obtiene el grado de calidad de la ejecución de las pruebas (velocidad de anticipación, coor- dinación bimanual, tiempos de reacciones múltiples).

Además, es importante considerar la información paralela que nos da la reali- 
zación de las pruebas, como el nivel de atención, concentración y comprensión, o la presencia de alteraciones de conducta, características por ejemplo de estados de nerviosismo. Se considerará normal o alterada, solo en el segundo caso se tomará nota de las puntuaciones directas o de los percentiles.

INTELIGENCIA PRÁCTICA (solamente en obtenciones)

\begin{tabular}{|l|l|l|}
\multicolumn{1}{l}{ Normal } & Alterada \\
\hline Test Inteligencia Práctica & & \\
\hline
\end{tabular}

La valoración de la inteligencia práctica,

obtención del permiso de conducir, y en los es necesaria siempre en aspirantes a la casos en que la anamnesis nos lo sugiera.

\section{TRASTORNOS MENTALES Y DE CONDUCTA}

\begin{tabular}{|l|l|l|}
\hline \multicolumn{1}{l}{ Normal Alterada } \\
\hline Escala Ansiedad- Depresión & & \\
\hline
\end{tabular}

Pretende determinar la existencia de malestar psicológico, que nos induzca a pensar en la presencia de algún trastorno mental o de conducta. Se realizará mediante tests de cribaje, de rápida ejecu- ción y corrección sencilla. Utilizamos generalmente el Test de Ansiedad y Depresión. En conductores profesionales y ante la sospecha de trastorno psicológico utilizamos el Cuestionario de Salud de Goldberg.

\section{EXPLORACIÓN DE ÁREAS COGNITIVAS}

\begin{tabular}{|l|l|l|}
\multicolumn{1}{c}{ Normal } & \multicolumn{1}{c|}{ Alterada } \\
\hline Prueba detección deterioro cognitivo & & \\
\hline
\end{tabular}

Se exploran las áreas cognitivas, en mayores de 70 años, y/o a cualquier edad, ante la sospecha de deterioro cognitivo, mediante el Test del Reloj (en primera instancia), seguido del Mini-Examen Cognoscitivo o el Test de Bender. El criterio de normalidad será el que nos indiquen los test.

Ante la sospecha de alguna alteración psicológica se realizarán anamnesis y exploración complementarias.

\section{- EXPLORACION ESPECÍFICA COMPLEMENTARIA DE PSICOLOGIA}

- ANAMNESIS COMPLEMENTARIA

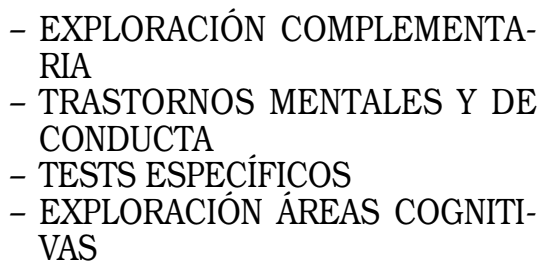

\section{ANAMNESIS COMPLEMENTARIA}

Nos amplia la información sobre el desarrollo de la vida actual del sujeto, contempla aspectos más concretos que pueden indicar la presencia de algún problema de tipo psicológico, y además nos informa de la influencia que ciertas alteraciones pueden tener sobre el individuo. 


\section{EXPLORACIÓN DE ÁREAS COGNITIVAS}

\begin{tabular}{|l|l|l|}
\hline ¿Tiene dificultades de concentración o memoria? & S & N \\
\hline ¿Tiene dificultad para conciliar el sueño, despierta fácilmente & S & N \\
\hline ¿Siente fatiga o cansancio excesivos? & S & N \\
\hline ¿Sufre dolor de cabeza o espalda? & S & N \\
\hline ¿Ha sentido palpitaciones o mareos? & S & N \\
\hline ¿Se siente observado o perseguido? & S & N \\
\hline ¿Se preocupa fácilmente? & S & N \\
\hline ¿Tiene cambios de humor frecuentes? & S & N \\
\hline ¿Se siente irritado o de mal humor con frecuencia? & S & N \\
\hline ¿Que actividades extralaborales realiza? & S & N \\
\hline ¿Está satisfecho de sus relaciones sociales? & S & N \\
\hline
\end{tabular}

\section{EXPLORACIÓN COMPLEMENTARIA}

Si se detecta alteración en el Test de Ansiedad y Depresión, para determinar la existencia de malestar psicológico, se realizará el Cuestionario de Salud de Goldberg.

Si se considera necesario y ante la sospecha de la existencia de algún trastorno, se podrán realizar tests específicos (de ansiedad, depresión, etc.).

Ante la sospecha de deterioro cognitivo observado mediante el test del Reloj se exploraran las áreas cognitivas mediante el Mini-Examen Cognoscitivo o el test de Bender.

\section{TRASTORNOS MENTALES Y DE CONDUCTA}

\begin{tabular}{|l|l|l|}
\multicolumn{2}{c}{ Normal } & Alterada \\
\hline Cuestionario de Salud de Goldberg & & \\
\hline
\end{tabular}

\section{TESTS ESPECÍFICOS}

\begin{tabular}{|l|l|l|}
\hline Inventario Situaciones Ansiedad en Tráfico ISA & & \\
\hline $\begin{array}{l}\text { Inventario Situaciones y Respuestas de Ansiedad } \\
\text { ISRA }\end{array}$ & & \\
\hline Inventario de Depresión de Beck BAI & & \\
\hline $\begin{array}{l}\text { Inventario de Indicadores Psicofisiológicos de } \\
\text { Cautela }\end{array}$ & & \\
\hline Cuestionario de Temores de Wolpe & & \\
\hline
\end{tabular}

\section{EXPLORACIONES COGNITIVAS}

\begin{tabular}{|l|l|l|}
\multicolumn{2}{c}{ Normal Alterada } \\
\hline Mini-Examen Cognoscitivo & & \\
\hline Test de Bender & & \\
Test del Reloj de Schulman & & \\
\hline
\end{tabular}




\section{EXPLORACIÓN DE LA INTELIGENCIA.}

En el caso de deterioro intelectual leve, se valorará la capacidad de aprendizaje y adaptación (en este caso son de gran utilidad las pruebas de aptitud perceptivo-motora), su capacidad en inteligencia práctica, imprescindible en la conducción, y si mantiene un nivel suficiente de comprensión, uso del lenguaje y memoria, necesarios para realizar el periodo de preparación en autoescuela.

El conocimiento de su historia escolar, nivel alcanzado, educación especial, asî como del grado de minusvalía, si la tiene, serán de espacial interés, para determinar el resultado de la exploración.

Las pruebas recomendadas para la exploración de estas áreas son:

- B-101 Inteligencia práctica de Bonnardell, libre de influencia cultural. Manipulación no verbal. Construcción de cubos.

- Escala de Alexander. Inteligencia práctica y libre de influencia cultural. Manipulación no verbal. Construcción de cubos.

- Test de Matrices Progresivas de Raven. Capacidad deducción de relaciones . Inteligencia general. Manipulación no verbal.

En ciertos casos, en comunicación con la autoescuela, se puede permitir que un alumno tenga la oportunidad de adaptarse y aprender las tareas necesarias para la realización del examen y las relacionadas con la conducción. Se considerará la posibilidad de limitación de la velocidad y/o del radio de conducción.

\section{EXPLORACIÓN DE LA APTITUD} PERCEPTIVO MOTORA

La aptitud preceptivo-motora se evalúa con los equipos psicotécnicos normalizados (instrumentos informatizados) con que cuentan los CRC

Se considerará al mismo tiempo que la calidad de la ejecución, la comprensión de instrucciones, la atención y la actitud al realizar las pruebas. Se comparará el déficit con posibles alteraciones detectadas en otras pruebas de la exploración, tanto médica como psicológica.

En caso de duda se realizará un "retest", en el que se valorará la capacidad de aprendizaje y de adaptación a las pruebas, junto a la calidad en la ejecución. Será de gran importancia la prueba de Tiempo de Reacciones Múltiples Discriminativas.

La solicitud de pruebas de conducción es recomendable en determinadas situaciones en las que persisten dudas sobre la aptitud del individuo. Sin embargo, la complejidad operativa de la ejecución de dichas pruebas hace difícil su realización.

Si la calidad en la ejecución no es totalmente satisfactoria, pero los resultados en conjunto son aceptables, se limitará la vigencia del permiso por un año 0 , excepcionalmente, otras limitaciones o restricciones, como la limitación de la velocidad y/o del radio de conducción.

Si la información obtenida a través de los pasos anteriores no resulta suficiente para determinar la aptitud perceptivo motora para conducir, la normativa ofrece la posibilidad de solicitar un informe complementario externo al CRC. 


\section{INFORME EXTERNO AL CENTRO}

Se solicitará ante la necesidad de ampliar información cuando los datos de la exploración básica y específica (complementaria) no son suficientes para determinar la aptitud del conductor.

\section{CONCLUSIONES}

las variables consideradas en la valoración de la aptitud perceptivo motora a lo largo de la historia, han servido a los modelos preventivos vigentes en cada época.

Los aspectos valorados, apenas han sufrido variaciones, en los últimos años. El sistema de evaluación y registro de datos evolucionó con la normalización de los aparatos psicotécnicos en 1988, permaneciendo sin modificaciones hasta la actualidad.

Las variables que han ido valorándose son: atención-concentración, velocidad de anticipación, coordinación bimanual vIsomotora, tiempo de reacción simple, tiempos de reacciones múltiples, inteli- gencia, capacidad de aprendizaje psicomotor, personalidad y estilos cognitivos

La utilización de protocolos de actuación que sigan el esquema expuesto en el presente artículo puede ayudar a unificar los criterios, de actuación profesional, con el fin de facilitar la uniformidad de las valoraciones de la aptitud perceptivo motora en los CRC.

Los criterios de actuación plasmados en este documento pueden ser utilizados, cuando sea necesario determinar la aptitud para conducir ante actuaciones contradictorias, o dispares en la determinación de la aptitud para conducir.

Donde la normativa vigente, deja a criterio facultativo la determinación de la aptitud y/o los condicionantes con los que debe otorgarse el permiso de conducir, el seguimiento de los protocolos estará especialmente indicado.

Los protocolos de valoración, permiten una valoración individualizada, ajustada a la normativa.

\section{BIBLIOGRAFÍA}

Varios (2002). Protocolo de Exploración MédicoPsicológica en centros de reconocimiento de conductores. Guía para la historia clínica básica. Dirección General de Tráfico, Madrid.

Varios (2003). Protocolo de Exploración Específica Médico-Psicológica en centros de reconocimiento de conductores. Guía para la Historia Clinica Específica. Dirección General de Tráfico, Edita: Sanidad y ediciones, S. L. Madrid.

Varios (2007). Protocolo de Exploración médico-psicológica para Centros de Reconocimiento de Conductores. Ministerio de Sanidad-Dirección General de Tráfico. Madrid 2007

Montoro L. Alonso F. Esteban C. Toledo F. (2000). Manual de Seguridad Vial. El factor humano. Barcelona.

Ozcoidi M. (2005). Tesis Doctoral CD Evolución histórica de la valoración de los criterios de aptitud psicofísicos para conducir en España. ISBN: 84370-6130-X Dep. Legal: V-2839-2005.

Serra J. y Tortosa F.(2003). Una historia de la psicología de la seguridad vial en España. Valencia: Promolibro.

Tortosa,F. , Civera,C. y Pastor,J.C. (2001). Una historia para un perfil profesional. El psicólogo experto en seguridad vial. Revista de Historia de la Psicología: 22(3-4), 543-559.

Tortosa,F. y Montoro,L. (2002). La psicología aplicada a la selección de conductores. Cién años salvando vidas. Psicothema 14(4), 714-725

Tortosa,F., Barjonet, P., Civera, C. y Montoro,L. (2003). Una historia de la psicología del tráfico y el transporte en Europa. Anuario de Psicología: 34(3), 401-416. 